\title{
Ginsenoside Rg5 attenuates hepatic glucagon response via suppression of succinate-associated HIF-1 $\alpha$ induction in HFD-fed mice
}

\author{
Na Xiao ${ }^{1}$ Meng-Die Lou ${ }^{1}$ Yi-Tong Lu ${ }^{1} \cdot$ Le-Le Yang ${ }^{1}$ - Qun Liu ${ }^{1} \cdot$ Baolin Liu $^{2}$. \\ Lian-Wen $\mathbf{Q i}^{1,2} \cdot$ Ping $\mathrm{Li}^{1}$
}

Received: 13 December 2016 / Accepted: 8 February 2017 /Published online: 9 March 2017

(C) Springer-Verlag Berlin Heidelberg 2017

\begin{abstract}
Aims/hypothesis Ginsenosides regulate glucose homeostasis. This study investigated the effect of ginsenoside $\operatorname{Rg} 5(\operatorname{Rg} 5)$ on the hepatic glucagon response, focusing on the regulation of metabolism.

Methods Mice fed a high-fat diet (HFD) showed increased hepatic glucose production (HGP). We observed the effects of $\operatorname{Rg} 5$ on hepatic fatty acid oxidation and glucagon response. The regulation of phosphodiesterase (PDE) 4B by succinate was also investigated in hepatocytes.

Results Rg5 inhibited endogenous glucose production in HFD-fed mice. Rg5 reduced cyclic AMP (cAMP) accumulation and inhibited transcriptional regulation of phosphoenolpyruvate carboxykinase (PEPCK) and glucose-6-phosphatase (G6Pase) by dephosphorylation of the cAMP response element-binding transcription factor in the liver, demonstrating the inhibitory effect on hepatic glucagon response. HFD feeding increased succinate accumulation in the liver due to the reversal of succinate dehydrogenase activation and triggered hypoxiainducible factor- $1 \alpha$ (HIF-1 $\alpha)$ induction. Succinate prevented cAMP degradation by inactivating PDE4B, thereby increasing
\end{abstract}

Electronic supplementary material The online version of this article (doi:10.1007/s00125-017-4238-y) contains peer-reviewed but unedited supplementary material, which is available to authorised users.

Lian-Wen Qi

qilw@cpu.edu.cn

Ping Li

liping2004@126.com

1 State Key Laboratory of Natural Medicines, China Pharmaceutical University, 24 Tongjia Lane, Nanjing 210009, China

2 Clinical Metabolomics Centre, China Pharmaceutical University, 639 Longmian Road, Nanjing 211198, China
cAMP accumulation in response to glucagon. Knockdown of HIF- $1 \alpha$ with small interfering RNA diminished the effect of succinate, indicating that HIF- $1 \alpha$ was essential for succinate to inactivate PDE4B. Rg5 inhibited succinate accumulation in hepatocytes by combating fatty acid oxidation, and thus reduced cAMP accumulation by blocking succinate/HIF- $1 \alpha$ induction. $\mathrm{Rg} 5$ reduced HGP as a consequence of the inhibition of the glucagon response.

Conclusions/interpretation Succinate acted as a metabolic signal to enhance the hepatic glucagon response. Rg5 reduced hepatic succinate accumulation by combating fatty acid oxidation and attenuated the hepatic glucagon response by suppressing succinate/HIF- $1 \alpha$ induction, suggesting that succinate-associated HIF- $1 \alpha$ induction in hepatocytes might be a therapeutic target in the treatment of diabetes.

Keywords cAMP · Ginsenoside Rg5 - Glucagon - HIF- $1 \alpha$. Hypoxia-inducible factor- $1 \alpha \cdot$ PDE4B $\cdot$ Phosphodiesterase 4B $\cdot$ Succinate

$\begin{array}{ll}\text { Abbreviations } \\ \text { AMPK } & \text { AMP-activated protein kinase } \\ \text { cAMP } & \text { Cyclic AMP } \\ \text { CREB } & \text { cAMP response element-binding protein } \\ \text { G6Pase } & \text { Glucose-6-phosphatase } \\ \text { HFD } & \text { High-fat diet } \\ \text { HGP } & \text { Hepatic glucose production } \\ \text { HIF-1 } \alpha & \text { Hypoxia-inducible factor-1 } \alpha \\ \text { MAS } & \text { Malate/aspartate shuttle } \\ \text { PDE } & \text { Phosphodiesterase } \\ \text { PEPCK } & \text { Phosphoenolpyruvate carboxykinase } \\ \text { PKA } & \text { Protein kinase A } \\ \text { Rg5 } & \text { Ginsenoside Rg5 } \\ \text { SDH } & \text { Succinate dehydrogenase }\end{array}$


siRNA Small interfering RNA

TMZ Trimetazidine

\section{Introduction}

Glucagon and insulin are secreted, respectively, by pancreatic alpha and beta cells to precisely control blood glucose homeostasis. Since its discovery, insulin has been considered an indispensable part of diabetes management; thus, most studies and therapeutic strategies focus on beta cell dysfunction and insulin resistance [1]. However, the importance of glucagon in normal glucose homeostasis and in the diabetic phenotype is underestimated. In fact, insulin reduces postprandial hyperglycaemia, while the basal glucagon level accounts for up to $70 \%$ of glucose production during fasting [2]. Diabetic patients and animal models of diabetes have abnormal secretion not only of insulin but also of glucagon [3]. In diabetes an enhanced hepatic glucagon response is responsible for fasting hyperglycaemia [4]. Total beta cell destruction fails to induce diabetes in glucagon receptor knockout mice [5], indicating that glucagon dysregulation is the sine qua non of diabetes. Glucagon increases glycaemia by promoting hepatic glucose output. It is therefore proposed that suppression of hepatic glucagon response may provide therapeutic advantages in diabetes management [6]. Metformin is a glucose-lowering agent and its action is mainly mediated through the suppression of hepatic gluconeogenesis [7].

By binding to the $\mathrm{G}$ protein-coupled receptor, glucagon activates cellular cyclic AMP (cAMP)/protein kinase A (PKA) signalling and phosphorylates transcription factor cAMP response element-binding protein (CREB), leading to increased hepatic glucose output through transcriptional upregulation of phosphoenolpyruvate carboxykinase (PEPCK) and glucose-6-phosphatase (G6Pase). In the cascades of hepatic glucagon signalling, the second messenger cAMP is a key regulator located upstream. Intracellular cAMP is synthesised by adenylyl cyclase, while phosphodiesterases (PDEs) could prevent cAMP accumulation through degradation. AMP-activated protein kinase (AMPK) is shown to antagonise hepatic glucagonstimulated cAMP signalling via PDE activation [8]. By contrast, hepatic NFKB p65 activation increases cAMP accumulation by suppressing PDE3B activity, leading to an enhanced hepatic glucagon response [9]. These events indicate that PDE activity is involved in the regulation of the hepatic glucagon response.

Succinate is an important intermediate in the citric acid cycle and emerges as a metabolic signalling pathway in response to cellular stress. In tumours and macrophages, succinate induces hypoxia-inducible factor- $1 \alpha(\mathrm{HIF}-1 \alpha)$ and reprogrammes glucose metabolism by switching to glycolysis
[10]. Succinate is accumulated in the liver under hepatic ischaemia. High succinate content has been detected in the blood of diabetic individuals [11-13], but its role in the hepatic glucagon response remains unknown.

Ginseng root and ginseng-containing herbal formulas are widely used in traditional Chinese medicine for enhancing health. Ginsenosides, the major bioactive constituents of ginseng, have shown beneficial effects on metabolic homeostasis [14-16]. Ginsenoside $\operatorname{Rg} 5$ ( $\mathrm{Rg} 5)$ is a major saponin in steam-pretreated ginseng [17]. However, the involvement of Rg5 in the regulation of metabolic disorders remains largely unknown. Here, we investigated the effect of $\mathrm{Rg} 5$ on hepatic gluconeogenesis, focusing on the regulation of metabolic alterations. We observed that $\mathrm{Rg} 5$ reduced hepatic succinate accumulation by combating fatty acid oxidation, and reduced hepatic glucose production (HGP) by attenuating the glucagon response, establishing a previously unrecognised role of ginsenosides in the regulation of hepatic gluconeogenesis.

\section{Methods}

Reagents Rg5 (purity >95\%) was purchased from Jiangsu Yongjian Pharmaceutical Technology (Jiangsu, China). Dimethyl succinate, dimethyl malonate and aminooxyacetate were bought from Sigma-Aldrich (St Louis, MO, USA). Palmitate (Sinopharm, Shanghai, China) was dissolved in ethanol and then diluted before use. PX-478 (Crene, Taizhou, China) and reconstituted glucagon (Novo Nordisk, Bagsvaerd, Denmark) were purchased from their respective commercial sources.

Animals Male C57BL/6J mice (6 weeks old) were purchased from Yangzhou University (Yangzhou, China). The mice were housed in colony cages with $12 \mathrm{~h} / 12 \mathrm{~h}$ light/dark cycles and fed either a normal chow diet or a high-fat diet (HFD, D12492; Research Diets, New Brunswick, NJ, Canada) ad libitum with free access to water. Animal care and experiments were approved by the Animal Ethics Committee of China Pharmaceutical University. For the HFD study, the mice were fed an HFD for 4 weeks, after which they were administered $\mathrm{Rg} 550 \mathrm{mg} \mathrm{kg}^{-1}$ day $^{-1}$ or metformin $200 \mathrm{mg} \mathrm{kg}^{-1}$ day $^{-1}$ by gavage for another 2 weeks. Blood was collected after an overnight fast and metabolic variables were assayed with commercial kits (Jiancheng, Nanjing, China). Glucagon and insulin in the blood were determined using ELISA kits (USCN, Wuhan, China). Insulin sensitivity index was calculated according to the formula: insulin sensitivity index $=1 /$ [blood glucose $(\mathrm{mmol} / \mathrm{l}) \times$ blood insulin $(\mathrm{pmol} / \mathrm{l})]$. 
Glucose, pyruvate and glucagon tolerance tests After an overnight fast, the HFD-fed mice were administered oral glucose $2 \mathrm{~g} / \mathrm{kg}$, and blood was collected for a glucose tolerance test. For the pyruvate tolerance test, the fasted mice were injected with pyruvate $2 \mathrm{~g} / \mathrm{kg}$. For the glucagon tolerance test in normal mice, the fasted mice were given oral $\mathrm{Rg} 5$ $50 \mathrm{mg} / \mathrm{kg}$ or metformin $200 \mathrm{mg} / \mathrm{kg}$, and $1 \mathrm{~h}$ later injected with glucagon $2 \mathrm{mg} / \mathrm{kg}$. Blood was collected at regular intervals to assay glucose content.

Primary mouse hepatocytes Male C57BL/6J mice were anaesthetised and the isolated liver was digested with collagenase type IV by perfusion through the inferior vena cava at a rate of $3 \mathrm{ml} / \mathrm{min}$. The whole liver was then removed and the hepatocytes were extracted in Williams' Medium E (Sigma-Aldrich) with 10\% (vol./vol.) FBS. After filtering, the cells were resuspended and cultured in six-well plates for further treatment.

cAMP quantification and PKA assays The primary hepatocytes were stimulated with palmitate, glucagon or dimethyl succinate, as indicated in the figure legends. The treated cells or liver of HFD-fed mice were lysed in cell lysis buffer, and the supernatant fraction was harvested for assays of cAMP and PKA activity using commercial kits (Cell Signalling Technology, Danvers, MA, USA; Abcam, Cambridge, MA, USA, respectively).

Hepatic glucose output The primary mouse hepatocytes were maintained in DMEM with $10 \%$ FBS. After planting, the media were replaced with serum-free DMEM for $18 \mathrm{~h}$. After washing, the cells were incubated in glucose-free media supplemented with $10 \mathrm{mmol} / \mathrm{l}$ pyruvate, $100 \mathrm{nmol} / 1$ glucagon and $100 \mu \mathrm{mol} / 1$ palmitate alone or with $\mathrm{Rg} 5$ at given concentrations for $6 \mathrm{~h}$. The supernatant fraction was then collected for glucose analysis.

Detection of mitochondrial complex II activity The primary mouse hepatocytes were treated with Rg5 $10 \mu \mathrm{mol} / 1$ in the presence of palmitate for $8 \mathrm{~h}$, and mitochondria were isolated using a mitochondria isolation kit (Jiancheng). Protein $10 \mu \mathrm{g}$ was then collected to assay complex II activity using a commercial kit (Jiancheng).

Pimonidazole staining for hypoxia and histopathological examination The HFD-fed mice were injected i.p. with pimonidazole $60 \mathrm{mg} / \mathrm{kg}$. The isolated liver was dehydrated and embedded in paraffin and pimonidazole staining was performed using a Hypoxyprobe-1 Plus Kit (Hypoxyprobe, Burlington, MA, USA). For hypoxia detection in the primary mouse hepatocytes, pimonidazole $200 \mu \mathrm{mol} / \mathrm{l}$ was added to the cells before incubation with palmitate $100 \mu \mathrm{mol} / 1$. The image was visualised using a fluorescence microscope. Liver slices were stained with haematoxylin and eosin, according to standard protocols.

Measurement of succinate concentration The liver (10 mg) of HFD-fed mice or primary mouse hepatocytes were rapidly homogenised in $100 \mu \mathrm{l}$ ice-cold succinate assay buffer. After centrifugation, the supernatant fraction was collected for the succinate assay using a Succinate Colorimetric Assay Kit (Sigma-Aldrich), according to the manufacturer's instructions.

Succinate dehydrogenase activity assay The primary mouse hepatocytes were treated with the indicated agents for $8 \mathrm{~h}$. The cells were lysed in cell lysis buffer and centrifuged to collect the supernatant fraction. After protein quantification, cellular succinate dehydrogenase (SDH) activity was assayed using a commercial kit (Jiancheng), according to the manufacturer's instructions. Samples of liver (50 mg) of HFD-fed mice were rapidly homogenised in $1 \mathrm{ml}$ cell lysis buffer for the assay of SDH activity.

Measurement of lactate and $\mathrm{NADH} / \mathrm{NAD}^{+}$ratio The primary mouse hepatocytes were pretreated with the indicated agents followed by palmitate $100 \mu \mathrm{mol} / 1$ stimulation. After treatment, the supernatant fraction was used for lactate assay using a commercial kit (Jiancheng). The cells were collected and detected using an NAD/NADH Quantitation Kit (Sigma-Aldrich) (electronic supplementary material [ESM] Methods).

HepG2 cell transfection Human hepatocellular carcinomaderived HepG2 cells were transfected with HIF1A small interfering RNA (siRNA) using siRNA transfection reagent (Santa Cruz, Santa Cruz, CA, USA) and pcDNA3.1-HIF1A (GenePharma, Shanghai, China) using Lipofectamine 2000 DNA transfection reagent (Invitrogen, Eugene, OR, USA), according to the manufacturer's instructions (see ESM Methods).

Real-time quantitative PCR Total RNA was isolated from the liver of HFD-fed mice or treated primary mouse hepatocytes, and the levels of $P g c-1 \alpha$ (also known as Ppargcla), Pepck (also known as Pck1), G6Pase (also known as G6pc), Acadvl, Acaala and Pde4b mRNA were quantified by real-time PCR (see ESM Methods, ESM Table 1).

Western blots Liver of HFD-fed mice or cell protein extraction and immunoblotting (40 $\mathrm{gg}$ protein samples) followed standard procedures. Antibody (p-AMPK, PDE4B, p-CREB, CD36, HIF-1 $\alpha$, G6Pase, PEPCK) reactivity was detected by electrochemiluminescence and quantified by densitometry using IPP, version 6.0, software (Media 
Cybernetics, Silver Spring, MD, USA). Antibody suppliers are listed in ESM Table 2.

Statistical analysis Results are expressed as the mean \pm SD. The significance of differences was analysed using one-way ANOVA followed by the Bonferroni test. GraphPad Prism software, version 6.0 (GraphPad Software, San Diego, CA, USA), was used for statistical analysis. A $p$ value of $<0.05$ was considered statistically significant.

\section{Results}

Rg5-inhibited endogenous glucose production in HFD-fed mice HFD feeding increased fasting blood glucose and glucose intolerance in mice. $\mathrm{Rg} 5$ administration lowered fasting blood glucose and reversed glucose intolerance with improvement of insulin sensitivity (Fig. 1a-c). Increased blood glucagon was not affected (Fig. 1d), indicating that $\operatorname{Rg} 5$ lowered fasting blood glucose without affecting glucagon secretion (Fig. 1d). A pyruvate tolerance test is an indicator of endogenous glucose production, since pyruvate load provides the substrate to promote HGP through gluconeogenesis. The hyperglycaemic response to pyruvate was significantly higher in the HFD-fed mice than in the chow-fed mice, whereas this alteration was reversed by $\operatorname{Rg} 5$, demonstrating its inhibitory effect on HGP (Fig. 1e). Rg5 attenuated the hyperglycaemic response to a glucagon challenge in normal mice (Fig. 1f), suggesting that Rg5 inhibited endogenous glucose production by restraining the hepatic glucagon response. Metformin showed similar regulation to that of $\operatorname{Rg} 5$.
In addition, $\operatorname{Rg} 5$ and metformin downregulated the elevated levels of blood NEFA, triacylglycerol and total cholesterol and reduced body weight gain in HFD-fed mice (ESM Fig. 1).

Rg5-attenuated hepatic glucagon response Glucagon mediates hepatic gluconeogenesis through cAMP/PKA activation. $\operatorname{Rg} 5$ and metformin effectively reduced cAMP production and inhibited PKA activity in the liver of HFD-fed mice (Fig. 2a, b). Similarly, Rg5 inhibited glucagon-induced cAMP accumulation and PKA activation (Fig. 2c, d) in the hepatocytes, and reduced glucagonmediated HGP (ESM Fig. 2). Meanwhile, Rg5 and metformin increased AMP content and AMPK phosphorylation in the liver of HFD-fed mice (Fig. 2e, f). PDE4B is a member of the PDE family and acts to degrade cAMP. PDE4B expression in the liver of HFD-fed mice was lower than that in chow-fed mice. Rg5 and metformin preserved PDE4B protein expression, indicating their ability to promote cAMP degradation (Fig. 2g). In response to cAMP/PKA signalling, CREB transcriptionally regulates gluconeogenic target genes. In HFD-fed mice, Rg5 and metformin inhibited CREB activation by dephosphorylation (Fig. 2h), and reduced gene expression of Pgc-1 $\alpha$, Pepck and G6Pase (Fig. 2i). These results showed that $\mathrm{Rg} 5$ inhibited the hepatic glucagon response in HFD-fed mice.

Rg5-combated hepatic fatty acid oxidation We investigated whether $\operatorname{Rg} 5$ suppressed the hepatic glucagon response via regulation of fatty acid metabolism. CD36 is a membrane scavenger receptor mediating lipid uptake. HFD feeding increased CD36 expression. Lipid deposits in the hepatocytes were viewed by hepatocellular vacuolisation (Fig. 3a, b),
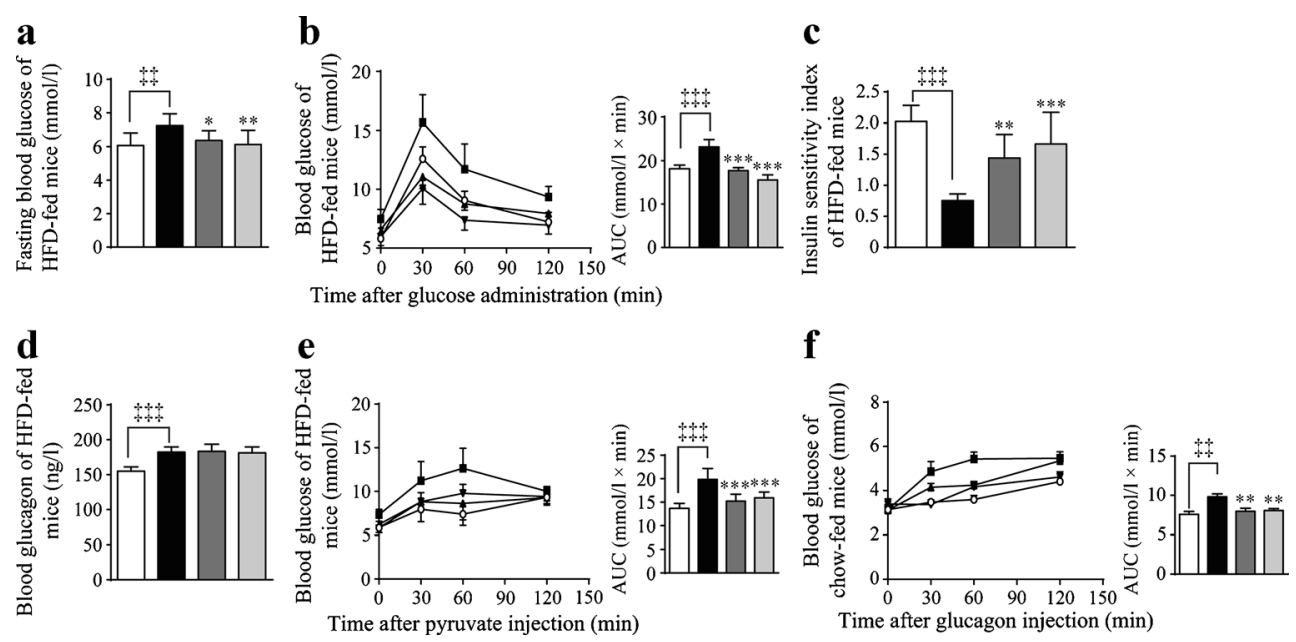

Fig. 1 Rg5-inhibited endogenous glucose production in HFD-fed mice. (a) fasting blood glucose levels; (b) blood glucose levels and AUC during the OGTT; (c, d) insulin sensitivity index and blood glucagon levels; (e) blood glucose levels and AUC during the pyruvate tolerance test; (f) blood glucose levels and AUC during the glucagon tolerance test in mice fed a chow diet. Data are expressed as mean $\pm \operatorname{SD}(n=10)$. White

bars, control; black bars, model; dark grey bars, Rg5; light grey bars, metformin $200 \mathrm{mg} / \mathrm{kg}$; white circles, control; black squares, model; upright black triangles, $\operatorname{Rg} 550 \mathrm{mg} / \mathrm{kg}$; inverted black triangles, metformin $200 \mathrm{mg} / \mathrm{kg} . * p<0.05, * * p<0.01$ and $* * * p<0.001$ vs model; $\$ p<0.01$ and $\$ p<0.001$ vs control 
Fig. 2 Rg5-attenuated hepatic glucagon response. (a, b) cAMP content and PKA activity in liver of HFD-fed mice; (c, d) cAMP content and PKA activity in primary mouse hepatocytes treated with glucagon $100 \mathrm{nmol} / \mathrm{l}$ (e-h) AMP content, p-AMPK, PDE4B and $p$-CREB expression in the liver of HFD-fed mice; (i) mRNA levels of Pgc-1 $\alpha$, Pepck and G6Pase in the liver $(\mathrm{Rg} 5$ $50 \mathrm{mg} / \mathrm{kg}$, metformin $200 \mathrm{mg} / \mathrm{kg}$ ). Data are expressed as mean $\pm \mathrm{SD}$ $(n=5-8)$. White bars, control; black bars, model; dark grey bars, Rg5; light grey bars, metformin. $* p<0.05, * * p<0.01$ and $* * * p<0.001$ vs model; ${ }^{\star} p<0.05$, $\star p<0.01$ and $\$ * p<0.001$ vs control. GAPDH, glyceraldehyde 3-phosphate dehydrogenase

Fig. 3 Rg5-combated hepatic fatty acid oxidation. (a) CD36 expression in the liver; (b) representative liver stained with haematoxylin and eosin $(\operatorname{Rg} 5$ $50 \mathrm{mg} / \mathrm{kg}$, metformin $200 \mathrm{mg} / \mathrm{kg}$; lipid deposit indicated by arrows; magnification $\times 400$ ); (c) mRNA levels of Acadvl and Acaala in hepatocytes treated with palmitate for $8 \mathrm{~h}$; (d) mitochondrial respiratory complex II activity in hepatocytes treated with palmitate for $8 \mathrm{~h}(n=3)$. Data are expressed as mean $\pm \operatorname{SD}(n=5)$. White bars, control; black bars, model; dark grey bars, Rg5; light grey bars, metformin; diagonally striped bars, TMZ. $* p<0.05, * * p<0.01$ and $* * * p<0.001$ vs model; $\$ p<0.01$ and ${ }^{* t} p<0.001$ vs control. GAPDH, glyceraldehyde 3-phosphate dehydrogenase
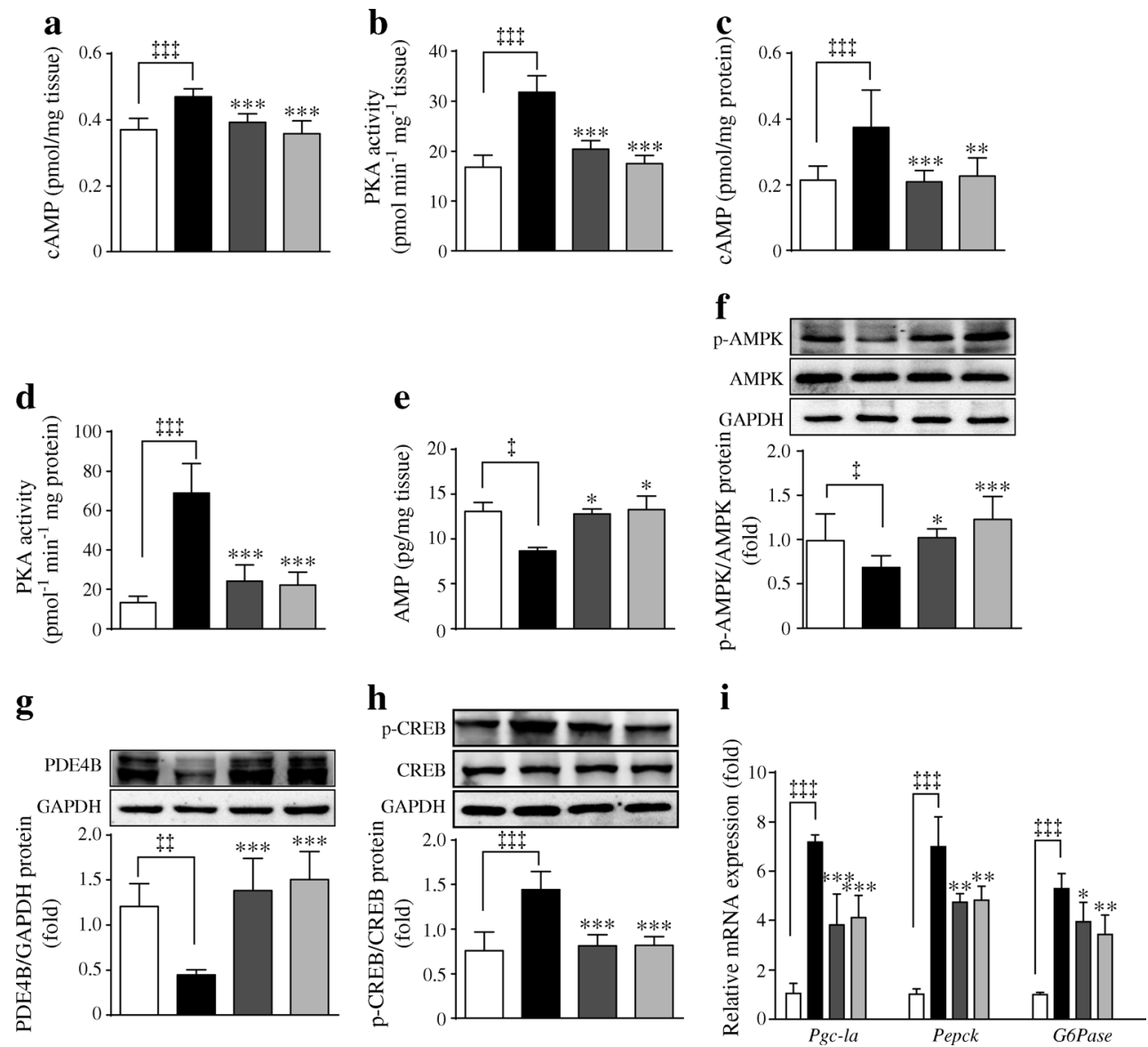

a
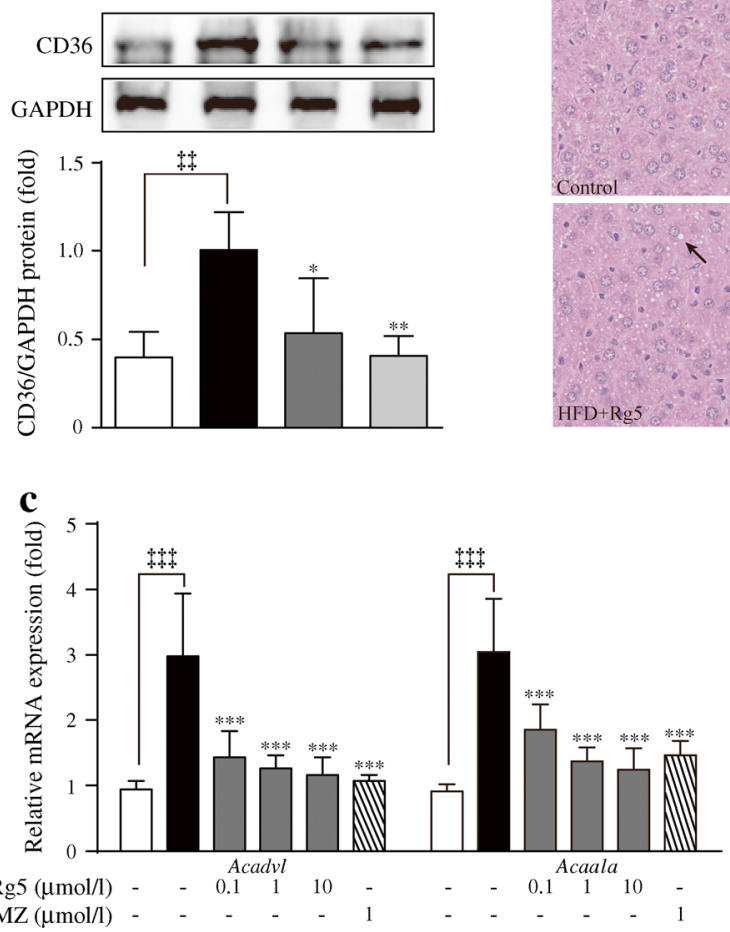

b

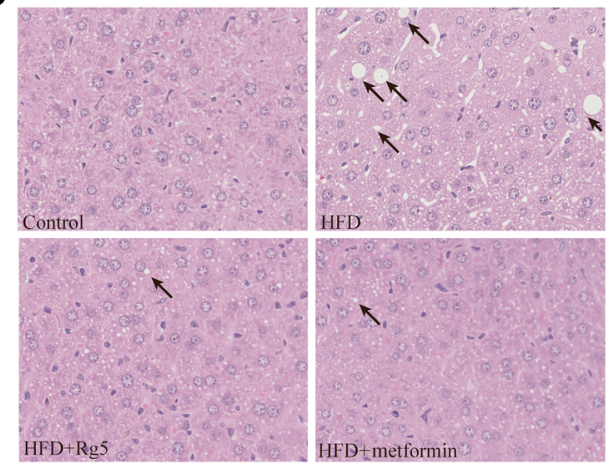

d

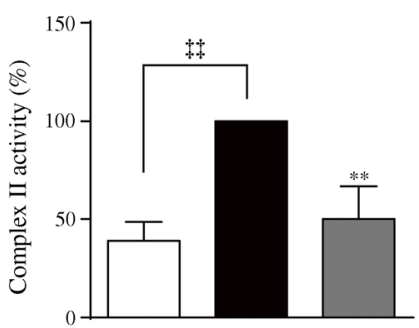


showing increased lipid entry into the liver. Rg5 and metformin inhibited lipid uptake by suppressing CD36 expression and, hence, reduced hepatic lipid accumulation (Fig. 3a, b). Fatty acyl CoA dehydrogenase (encoded by Acadvl) and 3-ketoacyl CoA thiolase (encoded by Acaala) are the key enzymes in $\beta$-oxidation, and their transcriptional expression is increased in palmitate-treated hepatocytes, indicative of enhanced fatty acid oxidation. Rg5 treatment normalised fatty acid oxidation by downregulating these gene expressions (Fig. 3c) and thus effectively inhibited mitochondrial respiratory chain complex II activity (Fig. 3d). Inhibiting fatty acid oxidation could reduce oxygen consumption. As expected, $\operatorname{Rg} 5$ and metformin prevented hepatic hypoxia in HFD-fed mice (ESM Fig. 3a). The Rg5 and $\beta$-oxidation inhibitor trimetazidine (TMZ) attenuated hypoxia in hepatocytes exposed to palmitate, further supporting our hypothesis (ESM Fig. 3b).

Rg5-reduced succinate accumulation in the liver In response to enhanced fatty acid oxidation, succinate accumulated with increased succinate dehydrogenase (SDH) activity in the liver of HFD-fed mice, whereas these alterations were reversed by Rg5 and metformin (Fig. 4a, b). Similarly, in the hepatocytes, $\operatorname{Rg} 5$ reduced palmitate-induced succinate production in a concentration-dependent manner (Fig. 4c). SDH converts succinate to fumarate; therefore, inhibition of SDH should increase succinate accumulation. However, in palmitate-treated hepatocytes, the SDH inhibitor dimethyl malonate reduced succinate production (Fig. 4c), indicating that palmitate-induced succinate accumulation might be due to the reversal of SDH activity. To confirm this, we examined SDH activity in the presence of palmitate and found that Rg5 inhibited palmitate-induced SDH activation in the hepatocytes (Fig. 4d). Amino-oxyacetate, an inhibitor of aspartate aminotransferase in the malate/aspartate shuttle (MAS), inhibited SDH activity, indicating that MAS activation contributed to the reversal of SDH activity and subsequent succinate production (Fig. 4d). MAS is a biochemical system for translocating electrons from the cytosol into the mitochondria. Palmitate treatment increased lactate accumulation and the NADH/NAD ${ }^{+}$ratio (Fig. 4e, f). The increased redox state should enhance MAS activity. $\operatorname{Rg} 5$ and TMZ lowered the cellular redox state by reducing lactate accumulation and the NADH/NAD ${ }^{+}$ratio (Fig. 4e, f). These results suggested that $\operatorname{Rg} 5$ lowered the cellular redox state by combating fatty acid oxidation and thereby preventing succinate production through reversal of SDH activity.

Succinate-mediated palmitate-induced PDE4B inhibition in hepatocytes To know whether succinate was involved in the action of palmitate to enhance the hepatic glucagon response, we examined PDE4B induction and cAMP production in the hepatocytes and found that palmitate
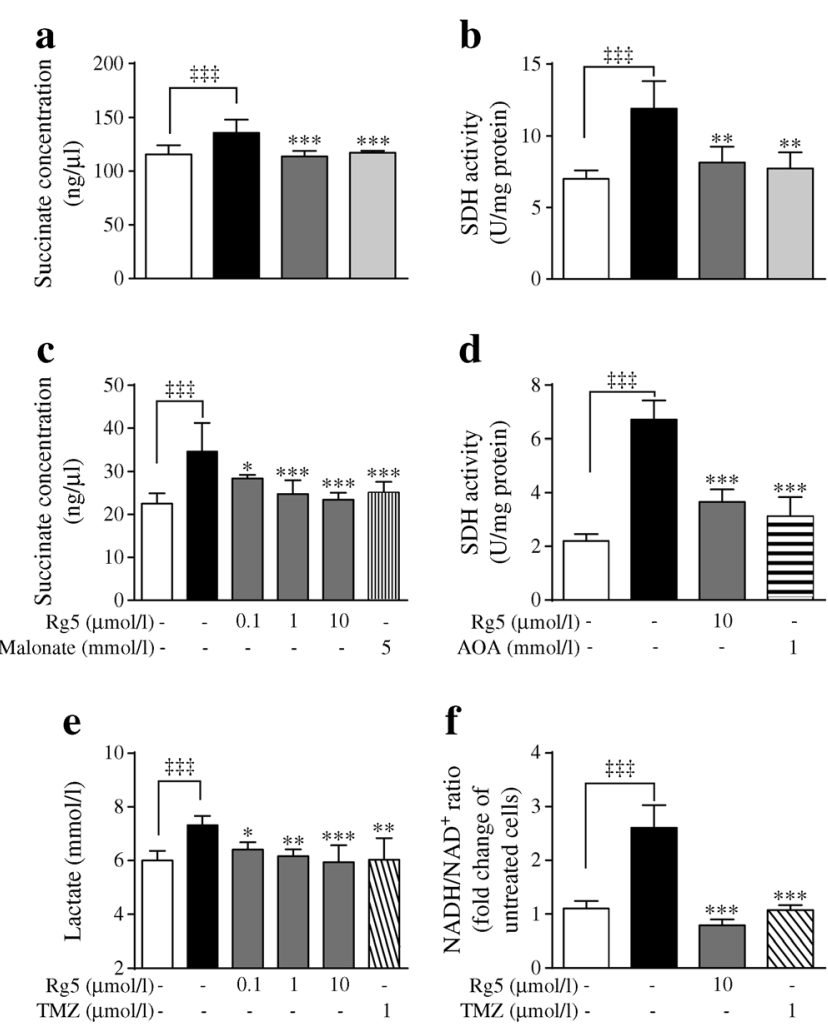

Fig. 4 Rg5-reduced succinate accumulation in the liver. (a, b) succinate accumulation $(n=8)$ and SDH activity $(n=4)$ in the liver of HFD-fed mice (Rg5 $50 \mathrm{mg} / \mathrm{kg}$, metformin $200 \mathrm{mg} / \mathrm{kg}$ ); (c, d) succinate accumulation and SDH activity $(n=4)$ in primary hepatocytes treated with palmitate $100 \mu \mathrm{mol} / \mathrm{l}$ for $8 \mathrm{~h}$; $(\mathbf{e}, \mathbf{f})$ lactate release and intracellular $\mathrm{NADH} / \mathrm{NAD}^{+}$ratio in hepatocytes treated with palmitate $100 \mu \mathrm{mol} / 1$ for 8 h. Data are expressed as mean $\pm \mathrm{SD}(n=6)$. White bars, control; black bars, model; dark grey bars, Rg5; light grey bars, metformin; vertically striped bars, dimethyl malonate (malonate); horizontally striped bars, amino-oxyacetate (AOA); diagonally striped bars, TMZ. ${ }^{*} p<0.05$, $* * p<0.01$ and $* * * p<0.001$ vs model; ${ }^{* * t} p<0.001$ vs control

stimulation reduced PDE4B expression and increased cAMP (Fig. 5a, b). Rg5 protected PDE4B expression against palmitate insult and thus prevented cAMP accumulation (Fig. 5a, b). Dimethyl malonate restored PDE4B expression and reduced cAMP production in palmitate-stimulated hepatocytes, indicative of the involvement of succinate (Fig. 5a, b). Treating the hepatocytes with cell-permeable dimethyl succinate consistently increased cAMP production in a condition-dependent manner (Fig. 5c). Moreover, dimethyl succinate promoted cAMP production in a manner similar to that of palmitate stimulation in the hepatocytes (Fig. 5d). These results suggested that succinate might act as a mediator in palmitate-induced cAMP accumulation.

Succinate-attenuated PDE4B expression dependent on HIF-1 $\alpha$ induction HFD feeding increased HIF- $1 \alpha$ accumulation in the liver, whereas this alteration was reversed by $\operatorname{Rg} 5$ and metformin (Fig. 6a). Similarly, Rg5 concentration-dependently inhibited palmitate-induced 
Fig. 5 Succinate-mediated palmitate-induced PDE4B inhibition in hepatocytes. $(\mathbf{a}, \mathbf{b})$ PDE4B expression $(n=5)$ and cAMP content in hepatocytes treated with palmitate $100 \mu \mathrm{mol} / 1$ for $8 \mathrm{~h}$; (c) cAMP content in hepatocytes treated with dimethyl succinate for $8 \mathrm{~h}$; (d) cAMP content in hepatocytes treated with palmitate or dimethyl succinate (circles, palmitate $100 \mu \mathrm{mol} / 1$; squares, succinate $5 \mathrm{mmol} / \mathrm{l})$. Data are expressed as mean $\pm \mathrm{SD}(n=6)$. White bars, control; black bars, model; dark grey bars, Rg5; vertically striped bars, dimethyl malonate (malonate). $* p<0.05, * * p<0.01$ and $* * * p<0.001$ vs model; ${ }^{\star} p<0.05,{ }^{*} p<0.01$ and $\$ p<0.001$ vs control. GAPDH, glyceraldehyde 3-phosphate dehydrogenase $\mathbf{a}$
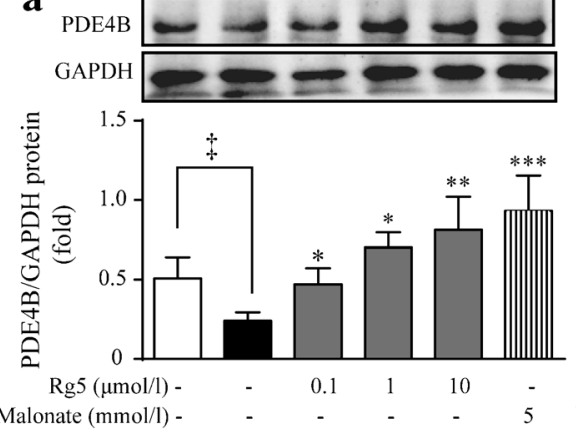

b

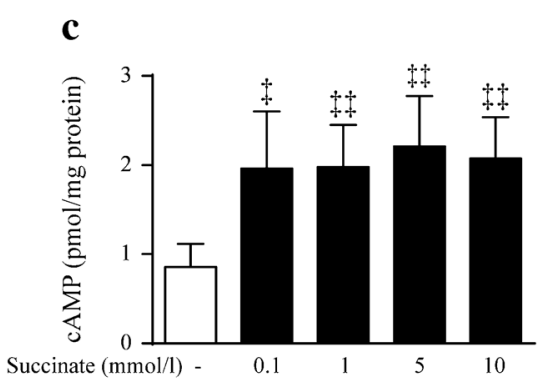

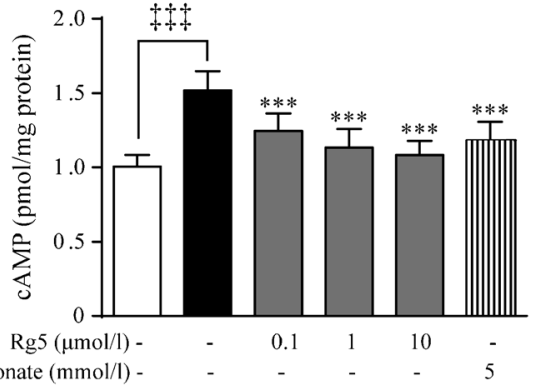

d

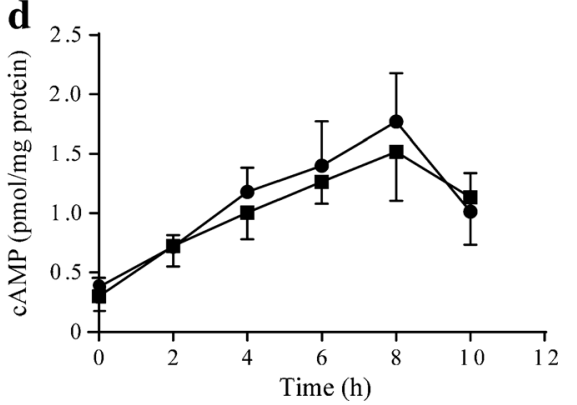

HIF- $1 \alpha$ induction in the hepatocytes (Fig. 6b). Dimethyl malonate attenuated palmitate-induced HIF- $1 \alpha$ expression, indicative of the role of succinate (Fig. 6b). Indeed, dimethyl succinate concentration-dependently induced HIF- $1 \alpha$ accumulation in the hepatocytes (Fig. 6c). These results indicated that succinate mediated palmitate-induced HIF- $1 \alpha$ induction. Dimethyl succinate reduced $P d e 4 b$ mRNA and PDE4B protein expression, whereas the HIF-1 $\alpha$ specific inhibitor PX-478 diminished the effect of succinate, indicating the potential role of HIF- $1 \alpha$ in succinate-mediated PDE4B suppression (Fig. 6d, e). We treated HepG2 cells with specific siRNA to silence HIFIA, and found that HIF- $1 \alpha$ knockdown diminished the inhibitory effects of palmitate and dimethyl succinate on PDE4B expression (Fig. 6f). Consistently, knockdown of HIF- $1 \alpha$ prevented palmitate and dimethyl succinate-induced cAMP accumulation in the hepatocytes (Fig. 6g). By contrast, HIF-1 $\alpha$ overexpression enhanced glucagon-induced cAMP accumulation in the hepatocytes (Fig. 6h). These results indicated that HIF-1 $\alpha$ was essential for palmitate or succinate-mediated PDE4B suppression.

\section{Rg5-attenuated glucagon-mediated HGP Palmitate} challenge increased the expression of glucagon-mediated G6Pase and PEPCK in the hepatocytes and further promoted HGP in response to glucagon (Fig. $7 \mathrm{a}-\mathrm{c}$ ). $\mathrm{Rg} 5$ treatment suppressed glucagon-induced G6Pase and PEPCK induction. This was more clearly observed when the glucagon response was enhanced by a palmitate challenge (Fig. 7a, b). As expected, Rg5 correspondingly reduced glucose output in response to glucagon (Fig. 7c). Together with the results mentioned above, our work showed that $\operatorname{Rg} 5$ prevented
cAMP accumulation by blocking succinate/HIF- $1 \alpha$ induction, and thus reduced HGP. The proposed pathway by which $\operatorname{Rg} 5$ restrains the hepatic glucagon response is shown in Fig. 7 d.

\section{Discussion}

Succinate acted as a metabolic signal to enhance the hepatic glucagon response. We showed that Rg5 blocked the succinate/HIF- $1 \alpha$ pathway by combating fatty acid oxidation, and reduced HGP by restraining the hepatic glucagon response, providing new insight into the role of $\operatorname{Rg} 5$ in the regulation of hepatic gluconeogenesis.

Glucagon increases hepatic glucose output through gluconeogenesis, while insulin counteracts the action of glucagon mainly at the transcriptional level [18]. Therefore, insulin resistance is tightly associated with an enhanced glucagon response in obesity and diabetes. The glucose tolerance test is an indicator of insulin sensitivity, because a glucose load induces insulin secretion to promote glucose disposal. Rg5 and metformin reversed glucose intolerance in the HFD-fed mice by improving insulin sensitivity, which should contribute to reducing HGP. Rg5 improved pyruvate tolerance in the HFD-fed mice and attenuated the hyperglycaemic response to glucagon, demonstrating its inhibitory effect on endogenous glucose production. Rg5 blocked glucagon-mediated cAMP/PKA activation, inactivated CREB by dephosphorylation, and consequently 

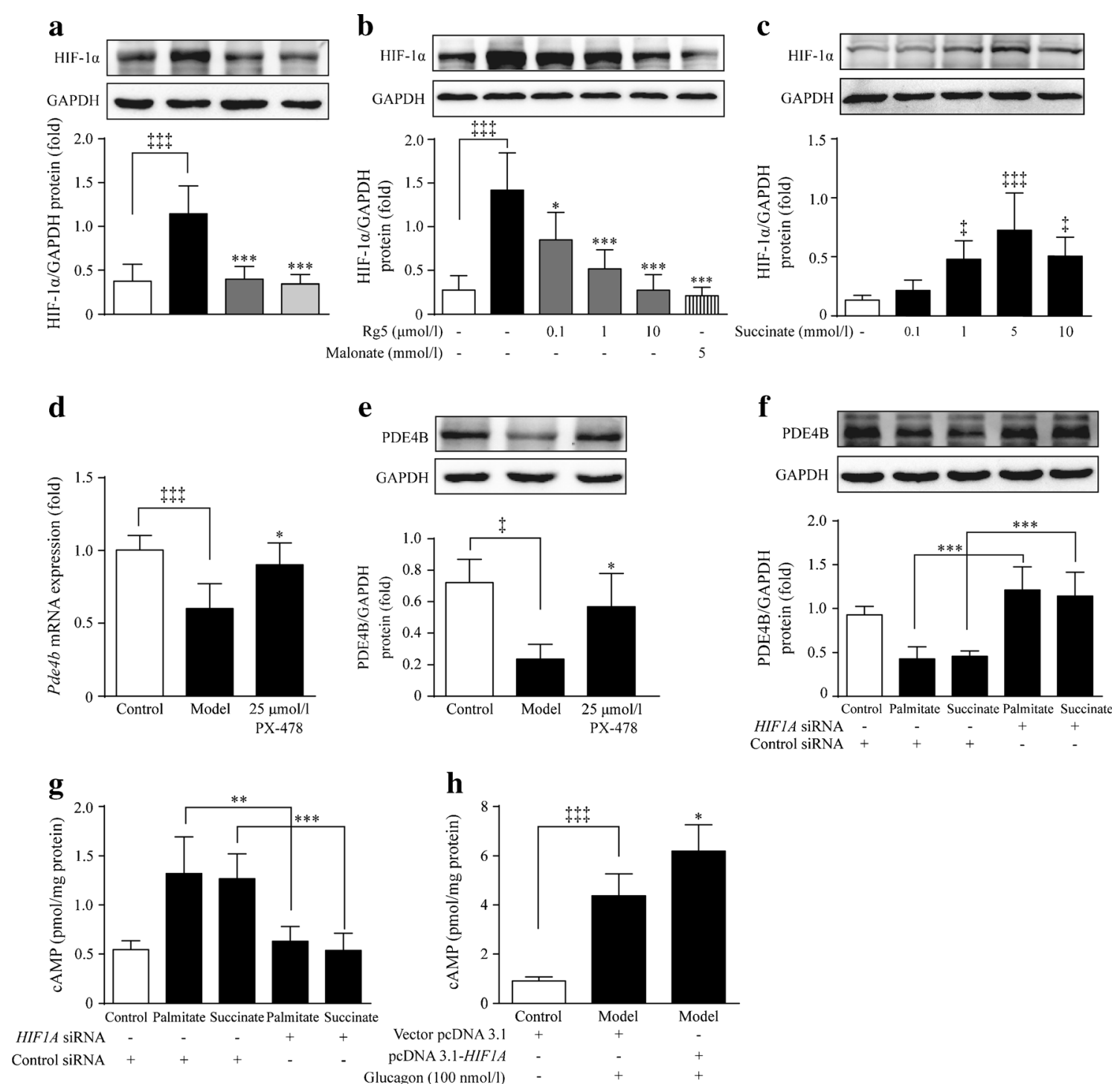

Fig. 6 Succinate-attenuated PDE4B expression dependent on HIF-1 $\alpha$ induction. (a) HIF-1 $\alpha$ expression in the liver of HFD-fed mice (Rg5 $50 \mathrm{mg} / \mathrm{kg}$, metformin $200 \mathrm{mg} / \mathrm{kg}$ ); (b) HIF-1 $\alpha$ expression in hepatocytes treated with palmitate $100 \mu \mathrm{mol} / \mathrm{l}$ or (c) dimethyl succinate (succinate). White bars, control; black bars, model; dark grey bars, Rg5; light grey bars, metformin, vertically striped bars, dimethyl malonate (malonate); (d, e) Pde $4 b$ mRNA and PDE4B protein expression in hepatocytes

treated with dimethyl succinate $5 \mathrm{mmol} / \mathrm{l}$ for $8 \mathrm{~h}$; (f, g) PDE4B expression and cAMP content $(n=6)$ in HepG2 cells transfected with HIF 1A siRNA; (h) cAMP content in HepG2 cells transfected with pcDNA3.1-HIF1A $(n=6)$. Data are expressed as mean $\pm \mathrm{SD}(n=5) . * p<0.05$, ${ }^{* *} p<0.01$ and $* * * p<0.001$ vs model; ${ }^{\star} p<0.05$ and ${ }^{+*} p<0.001$ vs control. GAPDH, glyceraldehyde 3-phosphate dehydrogenase

suppressed PEPCK and G6Pase induction, providing evidence to show that it reduced endogenous glucose production by suppressing the hepatic glucagon response.

In response to glucagon, cAMP is generated by adenylyl cyclase and acts as a second messenger to initiate glucagon signalling cascades in HGP. For more than 40 years adenosine nucleosides have been found to inhibit adenylyl cyclase activity [19]. Recently, metformin was shown to inhibit adenylyl cyclase activity and cAMP production by elevating the level of AMP, contributing to a decrease in HGP [20]. Rg5 increased AMP content in the liver of the HFD-fed mice, partially explaining its action in reducing cAMP accumulation. Increased AMP content could activate energy sensor AMPK; consistent with this, we found that both $\operatorname{Rg} 5$ and metformin increased AMPK phosphorylation in the liver of HFD-fed mice. Because AMP accumulation occurs before AMPK activation, it is impossible for $\mathrm{Rg} 5$ to regulate cAMP production via AMPK activation. However, AMPK is documented to act as a gluconeogenic suppressor to inhibit promoter activity of Pepck and G6Pase in hepatocytes $[21,22]$. Therefore, we speculated that AMPK activation by Rg5 could contribute to suppressing hepatic glucagon response in a manner that is independent of adenylyl cyclase and cAMP regulation. 
Fig. 7 Rg5-attenuated glucagon-mediated HGP. $(\mathbf{a}, \mathbf{b})$ glucagon-induced G6Pase and PEPCK protein expression in primary mouse hepatocytes treated with palmitate (PA) $(n=5)$. (c) HGP in response to glucagon in primary mouse hepatocytes treated with PA $(n=6) ;(\mathbf{d})$ the pathway depicting $\operatorname{Rg} 5$ restraint of hepatic glucagon response in the setting of lipid disorder. Data are expressed as mean \pm SD. $* p<0.05$ and $* * * p<0.001$ vs glucagon or model, ${ }^{\star} p<0.05$ and ${ }^{\star *} p<0.01$ vs control or glucagon. GAPDH, glyceraldehyde 3-phosphate dehydrogenase; AC, adenylyl cyclase $\mathbf{a}$
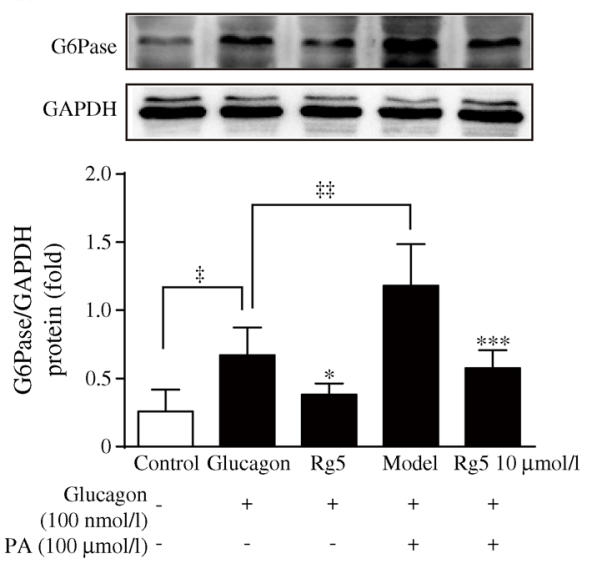

c

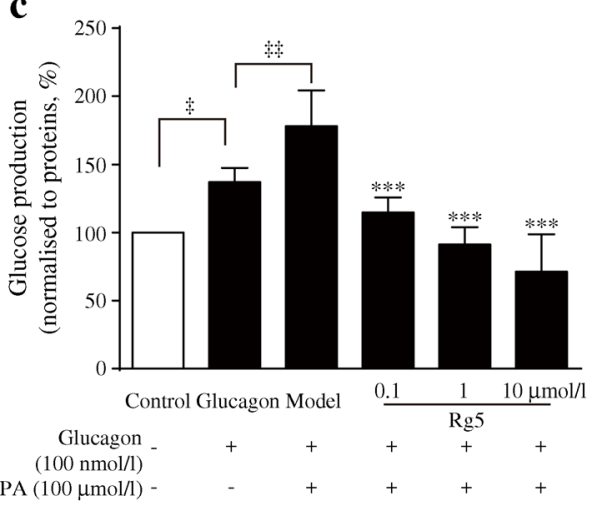

b
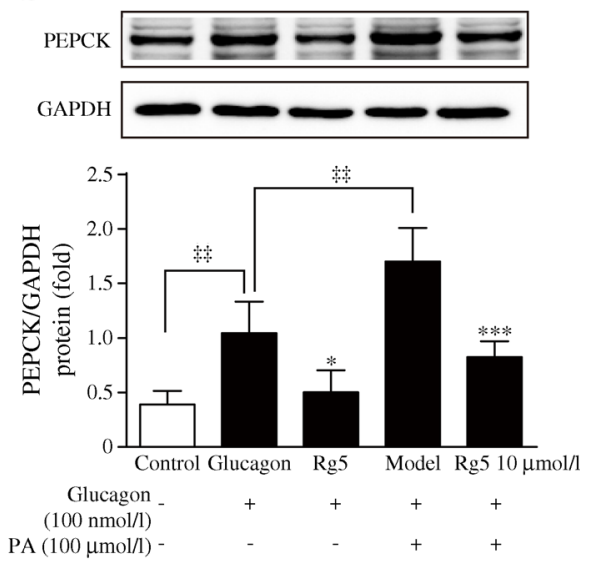

d

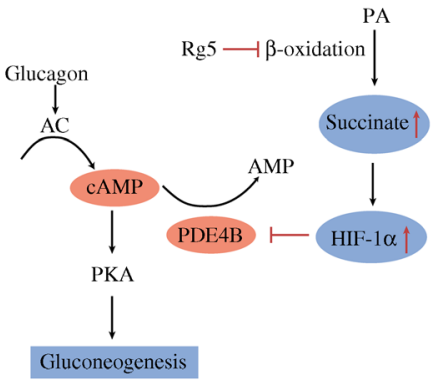

SDH conventionally operates in the citric acid cycle to convert succinate into fumarate; inhibition of SDH leads to intracellular succinate accumulation [23]. Interestingly, we found that succinate accumulated in the liver due to the reversal of SDH activity, in a manner similar to that of ischaemic accumulation of succinate in the heart [24]. HFD feeding increased lipid uptake in the hepatocytes and induced hepatic low oxygen tension, due to increased fatty acid oxidation. Consistent with this, saturated fatty acid is shown to induce adipose hypoxia by increasing oxygen consumption [25]. This alteration shifted cellular energy metabolism towards glycolysis, leading to lactate accumulation and an increased NADH/NAD ${ }^{+}$ratio. Therefore, the increased cytoplasmic NADH redox potential and the activation of MAS should be the driving force for succinate accumulation due to the reversal of SDH activity $[24,26]$. $\operatorname{Rg} 5$ decreased the cellular redox state by combating fatty acid oxidation, thereby reducing succinate accumulation in the hepatocytes.

As altered metabolism increased succinate accumulation, we wondered whether succinate acted as a mediator in the gluconeogenic response. Although PDE3/4 subfamilies are expressed in the liver $[9,27]$, PDE4B is thought to be the predominant $\mathrm{PDE}$ responsible for hepatic cAMP degradation $[8,20]$. The SDH inhibitor dimethyl malonate diminished the inhibitory effect of palmitate on PDE4B expression in the hepatocytes, suggesting that succinate might be an intermediate in the action of palmitate. Succinate stabilises and activates HIF- $1 \alpha$ by inhibiting prolyl hydroxylase in tumours [28]; it thus reprogrammes energy metabolism under anoxic conditions. Herein, we showed that, in the hepatocytes, HIF- $1 \alpha$ induction was essential for succinate to suppress PDE4B expression and prevent cAMP accumulation. Given that succinate accumulation was due to the altered cellular metabolism induced by enhanced fatty acid oxidation, it was reasonable to believe that succinate acted as a signalling mediator of palmitate activity to induce cAMP accumulation by suppressing PDE4B activation.

Rg5 inhibited succinate generation and prevented cAMP accumulation by preserving PDE4B activity. This regulation should contribute to suppressing the glucagon response in the liver. Indeed, $\mathrm{Rg} 5$ inhibited PEPCK and G6Pase expression in response to glucagon, and consequently reduced HGP in the hepatocytes. This regulation elucidated the underlying mechanism through which $\operatorname{Rg} 5$ inhibits hepatic gluconeogenesis in the setting of lipid disorders.

In conclusion, succinate acted as a metabolic signal to enhance the hepatic glucagon response. Rg5 reduced succinate accumulation by combating fatty acid oxidation 
and inhibited hepatic cAMP accumulation by blocking succinate/HIF-1 $\alpha$ signalling, contributing to restraint of the hepatic glucagon response. This study provides a novel therapeutic strategy for the prevention of hepatic gluconeogenesis in diabetes.

Acknowledgements We appreciate the editorial contribution of R. N. Alolga, a native English speaker at China Pharmaceutical University.

Data availability The data that support the findings of this study are available from the corresponding authors upon reasonable request.

Funding This work was supported in part by the National Natural Science Foundation of China $(81421005,81603353,91639115)$ and the Natural Science Foundation of Jiangsu Province (BK20160762).

Duality of interest The authors declare that there is no duality of interest associated with this manuscript.

Contribution statement L-WQ, PL and BL made substantial contributions to conception and design, acquisition, analysis and interpretation of data, as well as in drafting the article. NX, M-DL, Y-TL and L-LY made substantial contributions to acquisition, analysis and interpretation of data and reviewed the article critically for important intellectual content. QL made substantial contributions to analysis and interpretation of data and reviewed the article critically for important intellectual content. All authors approved the final version of the paper. L-WQ and PL are responsible for the integrity of this work.

\section{References}

1. Schwartz MW, Kahn SE (1999) Insulin resistance and obesity. Nature 402:860-861

2. Cherrington AD, Liljenquist JE, Shulman GI, Williams PE, Lacy WW (1979) Importance of hypoglycemia-induced glucose production during isolated glucagon deficiency. Am J Phys 236:E263E271

3. Jiang G, Zhang BB (2003) Glucagon and regulation of glucose metabolism. Am J Physiol Endocrinol Metab 284:E671-E678

4. Consoli A (1992) Role of liver in pathophysiology of NIDDM. Diabetes Care 15:430-441

5. Lee Y, Wang MY, Du XQ et al (2011) Glucagon receptor knockout prevents insulin-deficient type 1 diabetes in mice. Diabetes 60:391397

6. Unger RH, Cherrington AD (2012) Glucagonocentric restructuring of diabetes: a pathophysiologic and therapeutic makeover. J Clin Invest 122:4-12

7. Rena G, Pearson ER, Sakamoto K (2013) Molecular mechanism of action of metformin: old or new insights? Diabetologia 56:18981906

8. Johanns M, Lai YC, Hsu MF et al (2016) AMPK antagonizes hepatic glucagon-stimulated cyclic AMP signalling via phosphorylation-induced activation of cyclic nucleotide phosphodiesterase 4B. Nat Commun 7:10856

9. Ke B, Zhao Z, Ye X et al (2015) Inactivation of NF-kB p65 (ReLA) in liver improves insulin sensitivity and inhibits cAMP/PKA pathway. Diabetes 64:3355-3362
10. Mills E, O’Neill LA (2014) Succinate: a metabolic signal in inflammation. Trends Cell Biol 24:313-320

11. Correa PR, Kruglov EA, Thompson M, Leite MF, Dranoff JA, Nathanson MH (2007) Succinate is a paracrine signal for liver damage. J Hepatol 47:262-269

12. Hems DA, Brosnan JT (1970) Effects of ischaemia on content of metabolites in rat liver and kidney in vivo. Biochem J 120:105-111

13. Sadagopan N, Li W, Roberds SL et al (2007) Circulating succinate is elevated in rodent models of hypertension and metabolic disease. Am J Hypertens 20:1209-1215

14. Lee HM, Lee OH, Kim KJ, Lee BY (2012) Ginsenoside Rg1 promotes glucose uptake through activated AMPK pathway in insulinresistant muscle cells. Phytother Res 26:1017-1022

15. Yuan HD, Kim DY, Quan HY, Kim SJ, Jung MS, Chung SH (2012) Ginsenoside Rg2 induces orphan nuclear receptor SHP gene expression and inactivates GSK3beta via AMP-activated protein kinase to inhibit hepatic glucose production in HepG2 cells. Chem Biol Interact 195:35-42

16. Li W, Zhang M, Gu J et al (2012) Hypoglycemic effect of protopanaxadiol-type ginsenosides and compound $\mathrm{K}$ on type $2 \mathrm{di}$ abetes mice induced by high-fat diet combining with streptozotocin via suppression of hepatic gluconeogenesis. Fitoterapia 83:192198

17. Qi LW, Wang CZ, Yuan CS (2010) American ginseng: potential structure-function relationship in cancer chemoprevention. Biochem Pharmacol 80:947-954

18. Puigserver P, Rhee J, Donovan J et al (2003) Insulin-regulated hepatic gluconeogenesis through FOXO1-PGC-1alpha interaction. Nature 423:550-555

19. Fain JN, Pointer RH, Ward WF (1972) Effects of adenosine nucleosides on adenylate cyclase, phosphodiesterase, cyclic adenosine monophosphate accumulation, and lipolysis in fat cells. J Biol Chem 247:6866-6872

20. Miller RA, Chu Q, Xie J, Foretz M, Viollet B, Birnbaum MJ (2013) Biguanides suppress hepatic glucagon signaling by decreasing production of cyclic AMP. Nature 494:256-260

21. Shaw RJ, Lamia KA, Vasquez D et al (2005) The kinase LKB1 mediates glucose homeostasis in liver and therapeutic effects of metformin. Science 310:1642-1646

22. Kim YD, Park KG, Lee YS et al (2008) Metformin inhibits hepatic gluconeogenesis through AMP-activated protein kinase-dependent regulation of the orphan nuclear receptor SHP. Diabetes 57:306314

23. Tannahill GM, Curtis AM, Adamik J et al (2013) Succinate is an inflammatory signal that induces IL-1beta through HIF-1 alpha. Nature 496:238-242

24. Chouchani EE, Pell V, Gaude E et al (2014) Ischaemic accumulation of succinate controls reperfusion injury through mitochondrial ROS. Nature 515:431-435

25. Lee YS, Kim JW, Osborne $\mathrm{O}$ et al (2014) Increased adipocyte $\mathrm{O}_{2}$ consumption triggers HIF- $1 \alpha$, causing inflammation and insulin resistance in obesity. Cell 157:1339-1352

26. Barron JT, Gu L, Parrillo JE (1998) Malate-aspartate shuttle, cytoplasmic NADH redox potential, and energetics in vascular smooth muscle. J Mol Cell Cardiol 30:1571-1579

27. Abdollahi M, Chan TS, Subrahmanyam V, O’Brien PJ (2003) Effects of phosphodiesterase 3,4,5 inhibitors on hepatocyte cAMP levels, glycogenolysis, gluconeogenesis and susceptibility to a mitochondrial toxin. Mol Cell Biochem 252:205-211

28. Selak MA, Armour SM, MacKenzie ED et al (2005) Succinate links TCA cycle dysfunction to oncogenesis by inhibiting HIFalpha prolyl hydroxylase. Cancer Cell 7:77-85 